\title{
Review of: "RNA targeting with CRISPR-Cas13a facilitates bacteriophage genome engineering"
}

\author{
David Parker ${ }^{1}$ \\ 1 Cornell University
}

Potential competing interests: The author(s) declared that no potential competing interests exist.

Introduction and abstract are very strong. Premise of the paper seems well conceived and thoroughly researched. The use of an anti-CRISPR selection marker is unique and potentially quite profound. Methods are coherent and concise. I am a little bit skeptical of using a microplate for host range analysis, but the author offers a strong argument for why it might be useful to see the quality of the infection across multiple MOI. Data and analysis holds up the claims very well and figures are easy to understand. Great paper! 